\title{
Public health significance of mixed anxiety and depression: beyond current classification
}

\author{
Jayati Das-Munshi, David Goldberg, Paul E. Bebbington, Dinesh K. Bhugra, Traolach S. Brugha,
} Michael E. Dewey, Rachel Jenkins, Rob Stewart and Martin Prince

\section{Background}

The public health significance of mixed anxiety-depressive disorder (MADD) and the distinctiveness of its phenomenology have yet to be established.

\section{Aims \\ To determine the public health significance of MADD, and to compare its phenomenology with ICD-10 anxiety, depressive, and comorbid anxiety and depressive disorders. \\ Method \\ Weighted analysis of data from the Great Britain National Psychiatric Morbidity survey was conducted with a representative household sample of 8580 persons aged 16-74 years.}

\section{Results}

The 1-month prevalence of MADD was $8.8 \%$. A fifth of all days off work in Britain occurred in this group. The symptom profile of MADD was similar to 'pure' ICD-10 anxiety and depression, but with a lower overall symptom count. The disorder was associated with significant impairment of health-related quality of life. Differences in health-related quality of life measures between diagnostic groups were accounted for by overall symptom severity, which remained strongly associated with health-related quality of life measures after adjusting for diagnostic group. The finding that half of the anxiety, depression and MADD cases and a third of the comorbid depression and anxiety cases grouped into a single latent class challenges the notion of these conditions as having distinct phenomenologies. Mixed presentations may be the norm in the population.

\section{Conclusions}

The data support the pathological significance of MADD in its negative impact upon population health. Dimensional approaches to classification may provide a more parsimonious description of anxiety and depressive disorders compared with categorical approaches.

\section{Declaration of interest}

None.
Mixed anxiety-depressive disorder (MADD) is a provisional diagnosis in ICD-10 and DSM-IV, ${ }^{1,2}$ and describes the presence of sub-threshold depressive and anxiety symptoms. ${ }^{1-3}$ The notion that there might be a clinically relevant hinterland of mixed disorder beyond the specific anxiety and depression diagnoses is much older than current diagnostic nosology, ${ }^{3-5}$ and studies have reported groups of people with significant distress and impairment of functioning who do not meet diagnostic criteria for depression or anxiety. ${ }^{3}$ Mixed anxiety-depressive disorder remains 'subdefinitional' within both ICD-10 and DSM-IV, ${ }^{1,2}$ implying a lesser status within the diagnostic hierarchy. However, there is little information on the population-level impact of this disorder, compared with 'pure' or comorbid anxiety and depressive disorders. We therefore sought to investigate impairment in a variety of indicators of health-related quality of life, comparing them between those with pure ICD-10 depressive disorder, pure ICD-10 anxiety disorder, comorbid depressive and anxiety disorders, and MADD, using data from the Great Britain National Psychiatric Morbidity survey of $2000 .^{6}$ In addition, we sought to extend current debates regarding categorical $v$. dimensional approaches to the classification of the common mental disorders. Comorbidity has been argued to be a by-product of enforcing categorical constructs onto dimensions of symptoms. ${ }^{7-9}$ There is evidence to support the notion that 'cases' of psychiatric morbidity fall within a single spectrum of 'counts of common mental symptoms', with no evidence of individual or specific clustering of symptoms into disorders as proposed by existing diagnostic nosologies. ${ }^{10}$ Applying this dimensional model, MADD could be construed simply as the 'lower' end of a continuum where (subsyndromal) depression and anxiety symptoms coexist, whereas comorbid anxiety and depression might be at the 'higher' end of the continuum where 'diagnosable' depression and anxiety might coexist. We therefore set out to test, first, whether there is an independent effect of specific diagnostic categories (pure and comorbid anxiety and depressive disorders, and MADD) on health-related quality of life outcomes, after controlling for the effect of individual symptom counts, and, second, whether there is any evidence to support a distinctive psychopathological phenomenology for MADD, compared with 'pure' and comorbid anxiety and depressive disorders.

\section{Method}

\section{Setting}

The National Psychiatric Morbidity survey was conducted in 2000, and was the second in a series of government-sponsored surveys in Great Britain intended to monitor the prevalence, impact and treatment of common mental disorders, thereby informing policy and provision. Major design features are described here; full methodological details are available elsewhere. ${ }^{6}$

\section{Sample}

The study population comprised people aged 16-74 years living in private households in England, Scotland and Wales. The primary sampling units were 438 postcode sectors randomly selected from the Postcode Address File, stratified by region and socio-economic group. ${ }^{11}$ From each sampling unit, 36 addresses were selected at random. One eligible person was selected at random per household, using the Kish grid method. ${ }^{12}$ 


\section{Data collection}

Trained non-clinical interviewers carried out the initial computerassisted structured interviews. These were completed for 8580 persons, with a response rate of $69.5 \%$. The assessment of sociodemographic characteristics, impairment, use of services and neurotic psychopathology was made in a single interview of each participant, together with screening for psychosis and personality disorders, in a subsequent interview not considered further here.

\section{Measures}

The Clinical Interview Schedule - Revised (CIS-R) was used to assess for the presence of common mental disorders. ${ }^{13}$ The CIS-R includes 14 sections covering different symptom clusters: somatic symptoms, fatigue, concentration, sleep, irritability, worries over physical health, depression, depressive ideas, worry, anxiety, phobias, panic, compulsions and obsessions. Initial filter questions in each section establish the existence of a particular symptom in the previous month, leading to a more detailed assessment focusing on the past week. Symptom cluster sub-scale scores range from 0 to 4, except for the depressive ideas cluster sub-scale which has a maximum score of 5 . For each cluster 'clinically significant' symptoms are considered to be present if respondents score 2 or more on the relevant sub-scale. The 14 sub-scale scores are summed to create an overall CIS-R psychological morbidity score. Some further questions are included to enable ICD-10 diagnostic criteria to be applied using computer algorithms. Using this method, six ICD-10 diagnostic categories were obtained: obsessive-compulsive disorder, generalised anxiety disorder, depressive episode, phobias, panic and MADD. The last-named disorder was considered to be present if the participant scored 12 or more on the CIS-R overall psychological morbidity scale (considered as the optimal cut-off point for definition of clinically relevant morbidity), ${ }^{13}$ but did not fulfil criteria for any of the diagnoses elicited through ICD-10 diagnostic algorithms, as described above. Comorbidity was considered to be present if a participant simultaneously met ICD-10 criteria for any anxiety disorder and a depressive episode.

Alcohol use was assessed by means of the Alcohol Use Disorders Identification Test (AUDIT) ${ }^{14}$ This test was developed by the World Health Organization in order to identify people with hazardous or harmful patterns of alcohol use. Scores greater than 8 suggest problem drinking, with higher scores suggestive of harmful or hazardous alcohol use. For the purposes of this analysis, AUDIT scores were broken down into three groups: 0$8,8-15$ and $15-40$. Health-related quality of life was assessed using six impact indicators covering health, mental well-being and physical, social and occupational functioning:

(a) global health self-reported as 'poor';

(b) self-reported (lifetime) suicide attempt;

(c) poor physical functioning (scoring in the lowest fifth of the 12-item Short Form Health Survey (SF-12) Physical Component score; ${ }^{15}$

(d) impaired social and occupational functioning, defined as a 'yes' answer to SF-12 item 6, 'During the past 4 weeks, have you accomplished less than you would like with your work or other regular daily activities as a result of any emotional problems (such as feeling depressed or anxious)?;; ${ }^{15}$

(e) not currently employed (unemployed or economically inactive);

(f) 10 or more days off work in the previous year.

In addition, the following socio-demographic indicators were also recorded: age, gender, marital status and occupation.

\section{Statistical analysis}

Statistical analyses were conducted using Stata version 8.0 for Windows. Where possible, given the multistage stratified sampling design, analyses were weighted to take account of differing selection probabilities at each stage, and of non-response using post-stratification. Estimates of prevalence and association were made using the appropriate Stata survey commands to generate robust standard errors. The prevalence of the six key impairment indicators was estimated for those with MADD, pure depressive episode, pure anxiety disorder, and comorbid ICD-10 anxiety disorder with depressive episode, and for those with no mental disorder. Odds ratios were estimated for the associations between diagnostic group and impairment indicator comparing MADD (the reference category) with pure and comorbid ICD-10 anxiety disorders and depressive episodes. These crude estimates of association with health-related quality of life indicators were subsequently adjusted using the Stata weighted logistic regression svylogit procedure for age and gender, and then for age, gender and CIS-R psychological morbidity score (entered into the model as a continuously distributed variable estimating change in odds per unit increase in CIS-R score). Population attributable fractions for the associations between, first, diagnostic group (MADD, pure depression, pure anxiety, comorbid depression and anxiety, no diagnosis) and second, CIS-R psychological morbidity score in fifths, and each of the health-related quality of life indicators, were estimated using the Stata command aflogit from the prevalence ratios obtained from unweighted Poisson regression models controlling for age and gender. To investigate the grouping of participants in a data-driven way we carried out a latent class analysis. The R program (http://www.r-project.org) was used with the package poLCA. The 14 symptom cluster sub-scales, dichotomised as scores of less than 2 v. 2 or more, were used as the manifest variables. The optimal number of classes was determined using Akaike's information criterion. We report, for each class, the predicted class membership and the conditional item (symptom cluster) response probabilities.

\section{Results}

\section{Prevalence and socio-demographic correlates of common mental disorders}

The weighted prevalence rates for common mental disorders were depressive episode $2.6 \%$, panic disorder $0.7 \%$, generalised anxiety disorder $4.4 \%$, obsessive-compulsive disorder $1.1 \%$ and phobia $1.7 \%$. On this basis participants could be divided into three groups: depressive episode only (mild, moderate or severe depressive episode), weighted prevalence $1.1 \%(n=101)$; anxiety disorder only (generalised anxiety disorder, panic disorder, obsessive-compulsive disorder, phobia), 5.1\% $(n=485)$; and comorbid depressive and anxiety disorders, $1.5 \%(n=154)$. A further 769 participants (weighted prevalence 8.8\%) were categorised as having MADD on the basis of a score greater than 12 on the CIS-R and not meeting ICD-10 diagnostic criteria for any of the conditions listed above. Thus MADD was the most prevalent disorder, constituting around half of all common mental disorders. The remaining 7071 participants (weighted prevalence $83.6 \%$ ) were classified as 'non-cases' having no diagnosable neurotic mental disorder under the study criteria. The demographic characteristics of these five groups are described in Table 1. Participants with comorbid ICD-10 depression and anxiety were less likely to be married and more likely to be of lower occupational status. 
Table 1 Socio-demographic characteristics of sample by mental health status (weighted analysis)

\begin{tabular}{|c|c|c|c|c|c|}
\hline & Non-case & Depression & Anxiety & $\begin{array}{c}\text { Comorbid depression } \\
\text { and anxiety }\end{array}$ & $\begin{array}{l}\text { Mixed anxiety } \\
\text { and depression }\end{array}$ \\
\hline Female, \% & 48 & 68 & 58 & 50 & 61 \\
\hline Age, years: mean (s.d.) & $42.8(0.2)$ & $40.7(1.6)$ & $42.7(0.7)$ & $43.2(1.1)$ & $40.9(0.6)$ \\
\hline Married, \% & 64 & 50 & 54 & 43 & 58 \\
\hline Low-skilled occupation, \% & 20 & 21 & 26 & 35 & 15 \\
\hline
\end{tabular}

\section{Symptomatology}

Examination of the distribution of total CIS-R symptom scores (Fig. 1) indicates that those with comorbid ICD-10 anxiety and depressive disorders were most symptomatic, followed by those with ICD-10 depressive disorder, then by those with ICD-10 anxiety disorders, and then by those with MADD. All four diagnostic groups were markedly more symptomatic than the 'non-cases' group. The distribution of clinically significant symptoms (a score of 2 or more on each CIS-R symptom subscale) was similar in those with pure ICD-10 depressive disorder, pure anxiety disorder and MADD (Fig. 2), other than that those with depressive disorder who were more likely to have symptoms of depression and depressive ideas, and those with anxiety disorder who were more likely to have symptoms of anxiety and panic. Chi-squared tests performed for heterogeneity for each symptom (Fig. 2) by diagnostic group were $P<0.001$ in each case.

Within the group with a MADD diagnosis, $8.7 \%$ had neither specific symptoms of depression (depression or depressive ideas) nor anxiety (anxiety, panic or phobias); $24.3 \%$ had specific depression symptoms without specific anxiety symptoms; $19.1 \%$ had specific anxiety symptoms without depression symptoms; and $47.9 \%$ had both specific depression and anxiety symptoms. Non-specific symptoms predominated, with $98.9 \%$ experiencing one or more of somatic symptoms, fatigue, concentration problems, sleep problems, irritability or worry.

\section{Associations with quality of life indicators}

Next we tested for associations between the diagnostic groups and various indicators of health-related quality of life (Table 2). For

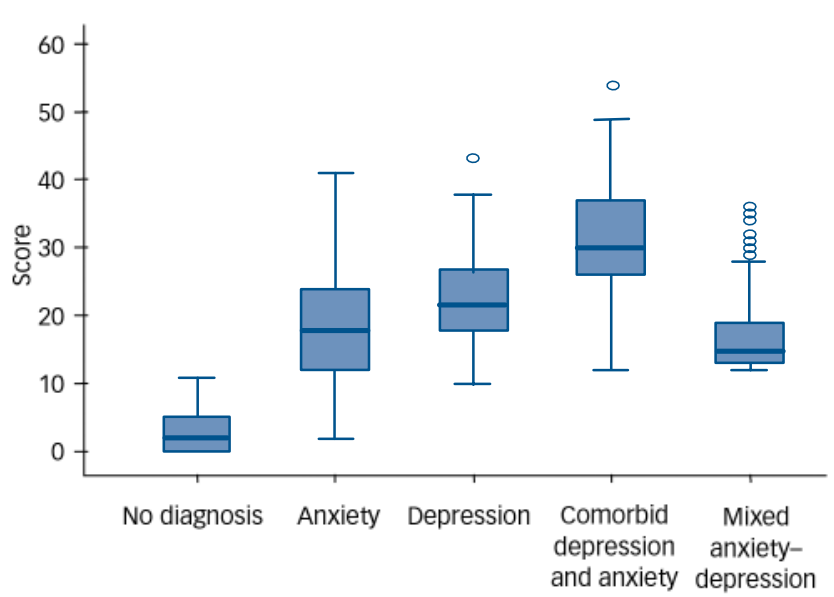

Fig. 1 Box-plot distribution of Clinical Interview Schedule - Revised total symptom scores for the five diagnostic groups (circles indicate outlier scores, more than 1.5 box widths from the median). each indicator, those categorised as non-cases were much less impaired than any of the four diagnostic groups. This was not the focus of this analysis and these results are not presented in more detail. Compared with participants with MADD, after adjusting for age and gender, those with comorbid ICD-10 depression and anxiety were more likely to report poor health, worse functioning, lifetime suicide attempts and unemployment. Those with anxiety disorder were also slightly more likely to report lifetime suicide attempts. No other group difference was found. Adjusting for comorbid alcohol use using the AUDIT did not affect these findings. However, much - if not all - of the effect of diagnostic group upon health-related quality of life indicators was fully accounted for after controlling for total number of symptoms (CIS-R psychological morbidity score). It was clear from the same models that there was an independent statistically significant effect of number of symptoms upon each of the healthrelated quality of life indicators after adjusting for diagnostic group, age and gender. The odds ratios per 1-point increase in CIS-R score were as follows: for poor health OR $=1.12(95 \% \mathrm{CI}$ 1.09-1.15); for suicide attempt $\mathrm{OR}=1.10$ (95\% CI $1.07-1.13)$; for the bottom fifth of the SF-12 Physical Component score $\mathrm{OR}=1.12$ (95\% CI 1.10-1.14); for 'health results in accomplishing less' OR=1.11 (95\% CI 1.09-1.14); for unemployment $\mathrm{OR}=1.05$ (95\% CI 1.03-1.07); and for days off work OR=1.08 (95\% CI $1.05-1.10)$. We compared directly the overall effect first of diagnostic group and then of number of symptoms upon each of

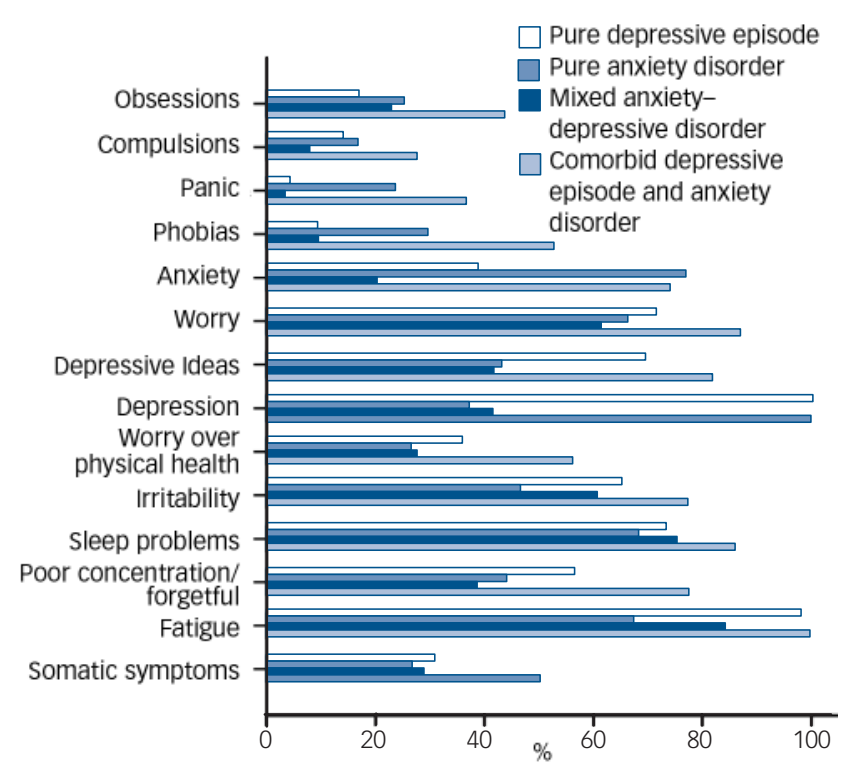

Fig. 2 Prevalence of clinically significant symptoms (two or more reported in each category) among those with pure depressive episodes, pure anxiety disorders, mixed anxiety-depressive disorder, and comorbid depressive episode and anxiety disorder. 
Table 2 Association of common mental disorder diagnoses and health-related quality of life indicators (weighted analysis)

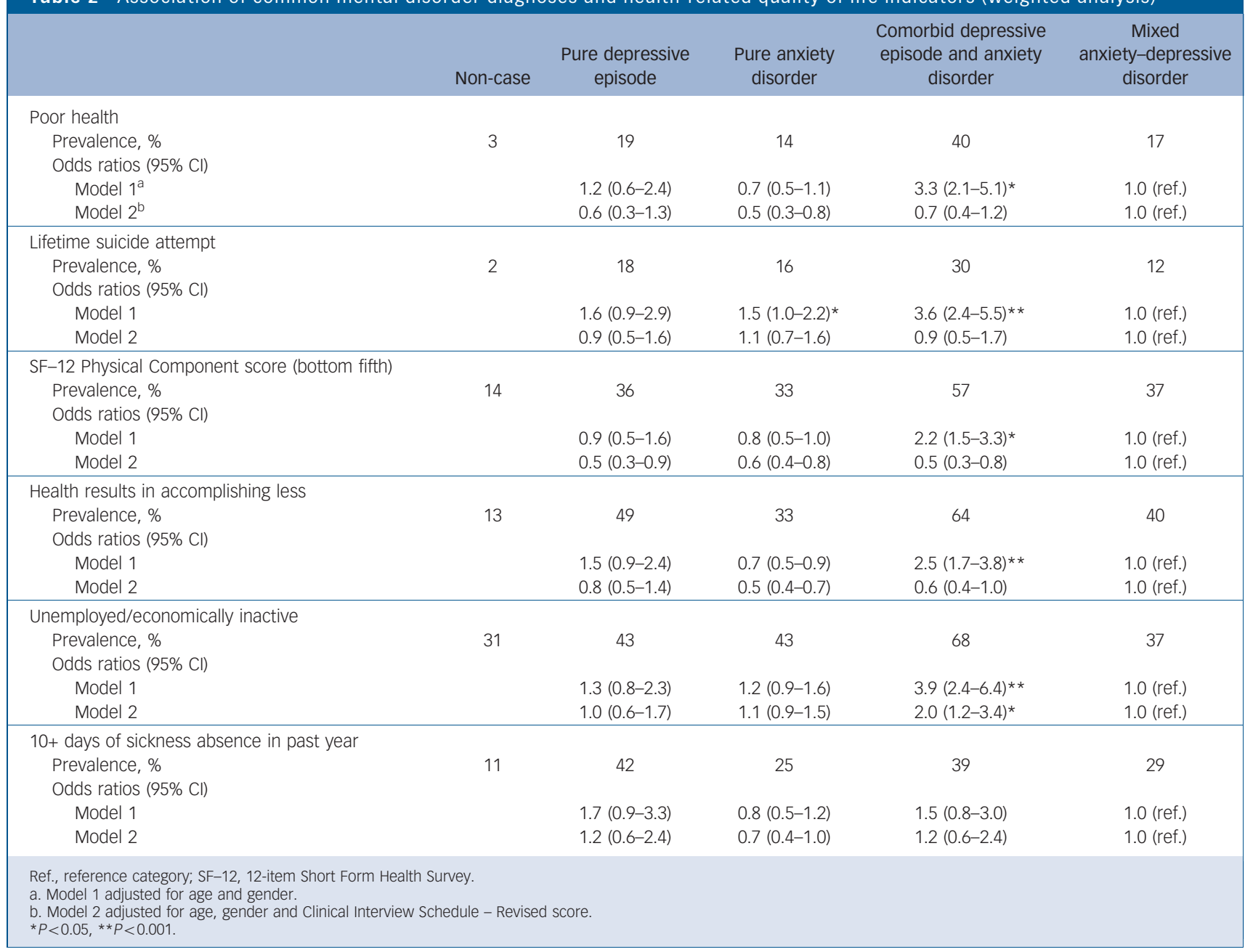

the health-related quality of life indicators using population attributable fractions (Table 3). For each indicator the total population attributable fraction for number of symptoms comfortably exceeded that for diagnostic group.

By weighting back to the base population, we were able to estimate the total days off work per annum contributed in Great Britain by people in five mutually exclusive categories: those categorised as 'non-cases' (i.e. no discernible common mental disorder) and those in the four mental health diagnostic groups. The results were as follows: non-case group, 120.7 million days (95\% CI 105.0-137.0) (i.e. $59 \%$ of the total 204 million days taken off work per annum); pure ICD-10 depression group, 7.2 million days (95\% CI 2.4-12.0) (i.e. $4 \%)$; pure ICD-10 anxiety disorder group, 25.1 million days (95\% CI 16.4-33.7) (i.e. 12\%); comorbid ICD-10 depression and anxiety disorder group, 9.6 million days (95\% CI 1.7-17.2) (i.e. 5\%); MADD group, 41.4 million days (95\% CI 28.6-54.1) (i.e. 20\%).

\begin{tabular}{|c|c|c|c|c|c|c|}
\hline & \multicolumn{6}{|c|}{ Population attributable prevalence fraction, \% $(95 \% \mathrm{Cl})^{a}$} \\
\hline & $\begin{array}{c}\text { Pure } \\
\text { depression }\end{array}$ & $\begin{array}{c}\text { Pure } \\
\text { anxiety }\end{array}$ & $\begin{array}{l}\text { Comorbid depres- } \\
\text { sive episode and } \\
\text { anxiety disorder }\end{array}$ & $\begin{array}{l}\text { Mixed anxiety- } \\
\text { depressive } \\
\text { disorder }\end{array}$ & $\begin{array}{l}\text { Total } \\
\text { PAF for } \\
\text { diagnoses }^{b}\end{array}$ & $\begin{array}{l}\text { Total } \\
\text { PAF for } \\
\text { symptoms }^{c}\end{array}$ \\
\hline Poor health & $2.0(1.7-2.3)$ & $7.9(7.1-8.8)$ & $5.2(5.0-5.5)$ & $13.1(12.1-14.2)$ & $28.3(26.6-30.0)$ & $59.8(55.2-63.8)$ \\
\hline Lifetime suicide attempt & $3.8(2.2-5.3)$ & $15.3(12.1-18.5)$ & $10.7(8.5-12.8)$ & $17.8(14.0-21.5)$ & $47.6(42.1-52.5)$ & $78.0(68.2-84.7)$ \\
\hline SF-12 Physical Component score (bottom fifth) & $0.8(0.6-1.0)$ & $3.9(3.4-4.4)$ & $2.3(2.1-2.5)$ & $7.5(6.9-8.1)$ & $14.5(13.6-15.6)$ & $45.3(41.8-48.6)$ \\
\hline Health results in accomplishing less & $2.3(1.9-2.7)$ & $6.3(5.3-7.3)$ & $4.7(4.3-5.1)$ & $13.2(12.0-14.5)$ & $26.5(24.6-28.3)$ & $64.1(59.4-68.3)$ \\
\hline Unemployed/economically inactive & $0.5(0.3-0.6)$ & $2.3(1.9-2.7)$ & $1.8(1.7-1.9)$ & $2.1(1.5-2.6)$ & $6.7(5.9-7.4)$ & $9.4(7.3-11.5)$ \\
\hline $10+$ days of sickness absence in past year & $2.6(1.8-3.4)$ & $6.3(4.5-8.1)$ & $11.1(8.9-13.2)$ & $3.5(2.3-4.8)$ & $23.5(20.2-26.7)$ & $44.8(36.3-52.1)$ \\
\hline \multicolumn{7}{|c|}{$\begin{array}{l}\text { PAF, population attributable fraction; SF-12, 12-item Short Form Health Survey. } \\
\text { a. Population attributable prevalence fractions adjusted for age and gender. } \\
\text { b. Pure depression, pure anxiety, comorbid depressive episode and anxiety disorder, mixed anxiety-depressive disorder. } \\
\text { c. Total Clinical Interview Schedule - Revised psychological morbidity scores in fifths. }\end{array}$} \\
\hline
\end{tabular}




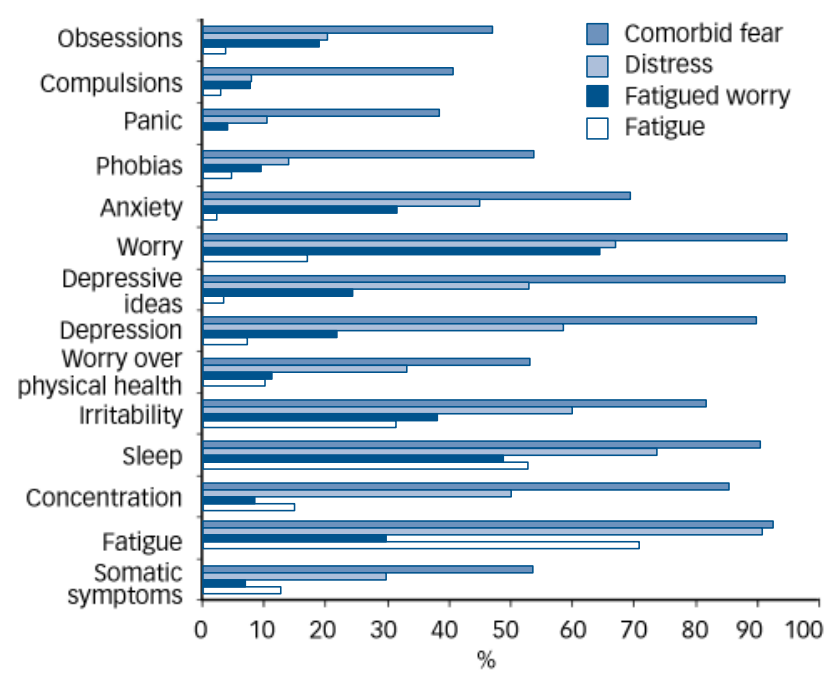

Fig. 3 Conditional probability of clinically significant symptoms (two or more symptoms reported in each category) by latent class.

\section{Latent class analysis}

Latent class analysis (LCA) of the data revealed five classes (Table 4). Figure 3 displays how each of the four main pathological classes (the fifth class, comprising mostly non-cases is not shown) is associated with the reporting of clinically significant symptoms in each symptom cluster. The first class ('comorbid fear' in Fig. 3) tended to represent cases previously diagnosed as comorbid depression and anxiety. The second class ('distress') included a significant proportion of all four of the original diagnostic categories ('pure' depression, 'pure' anxiety, comorbid depression and anxiety, and nearly half of all MADD cases). The third ('fatigued worry') and fourth ('fatigue') classes were dominated by participants who had been previously classified as 'non-cases', alongside a significant proportion of those previously diagnosed with MADD (Table 4). The fifth class appeared to be mostly represented by cases formerly within the non-case group.

\section{Discussion}

\section{Strengths and weaknesses}

This analysis used data from a nationally representative population survey with a large sample size. All participants were administered a structured clinical interview, the CIS-R, generating ICD-10 diagnoses. Unusually, the CIS-R includes a comprehensive assessment of anxiety and depressive symptoms regardless of whether the respondent meets criteria for a specific disorder. It is therefore particularly well suited for exploration of sub-threshold conditions such as MADD. The National Psychiatric Morbidity survey also incorporated multiple indicators of impairment covering different domains of health-related quality of life.

There are, however, some limitations. The CIS-R is a fully structured assessment administered by trained lay interviewers, and concerns have been expressed regarding the validity of such measures, particularly at the level of individual diagnoses. ${ }^{16,17}$ The MADD criterion developed by the National Psychiatric Morbidity survey investigators has not previously been validated, and did not map precisely on to those proposed in ICD-10. In addition, the cross-sectional design and reliance on self-reported outcomes make it difficult to exclude the possibility that information bias could have led to an overestimation of the association between common mental disorders and the various indices of impairment in health-related quality of life. The high frequency of non-responders $(31.5 \%)$ might have introduced a variety of biases with respect to prevalence and association with healthrelated quality of life indicators. The characteristics of nonresponders are unknown.

\section{Should MADD be considered a sub-definitional disorder?}

Our analyses of data from the National Psychiatric Morbidity survey suggest that MADD may account for half of all cases of common mental disorder in Great Britain. The impact of MADD upon health-related quality of life is similar to that of pure anxiety and depression, but somewhat less than that of comorbid disorders. Twelve per cent of those with MADD reported a lifetime suicide attempt. Twenty per cent of all disability days in Great Britain occurred in people with MADD, accounting for around half of all the disability days occurring in people with common mental disorders. The results of this analysis support the pathological significance of MADD, which does not seem to be a sub-definitional disorder at least in terms of its negative impact upon population health and well-being. This is an important consideration. Critics have rightly queried the tendency to extend the boundaries of what is considered mental disorder, arguing that this involves the medicalisation of normal human distress. However, our data suggest that many cases of MADD have merely slipped through the gaps in the current classificatory system. Once careful attention had been given to the diagnostic criteria (see below), inclusion of MADD would seem to be amply justified as a necessary correction to omissions in the current classification, rather than an attempt to lower the threshold to include minor cases of dubious psychopathological significance.

\section{Implications for phenomenology and classification}

That one latent class includes three-quarters of the pure depression cases, half of the anxiety cases and a third of the comorbid depression and anxiety cases challenges the notion of these conditions as having distinct phenomenologies, once the complete

\begin{tabular}{|c|c|c|c|c|c|c|}
\hline Latent class & $\begin{array}{c}\text { Non-case } \\
n\end{array}$ & $\begin{array}{l}\text { Pure } \\
\text { depressive } \\
\text { episode } \\
n\end{array}$ & $\begin{array}{c}\text { Pure anxiety } \\
\text { disorder } \\
n\end{array}$ & $\begin{array}{c}\text { Comorbid depressive } \\
\text { episode and } \\
\text { anxiety disorder } \\
n\end{array}$ & $\begin{array}{c}\text { Mixed } \\
\text { anxiety-depressive } \\
\text { disorder } \\
n\end{array}$ & $\begin{array}{c}\text { Class } \\
\text { membership } \\
\%\end{array}$ \\
\hline 1 & 0 & 15 & 64 & 98 & 23 & 2.6 \\
\hline 2 & 15 & 80 & 232 & 56 & 369 & 8.8 \\
\hline 3 & 408 & 2 & 142 & 0 & 154 & 9.4 \\
\hline 4 & 966 & 4 & 32 & 0 & 222 & 17.1 \\
\hline 5 & 5682 & 0 & 15 & 0 & 1 & 62.1 \\
\hline Total & 7071 & 101 & 485 & 154 & 769 & 100 \\
\hline
\end{tabular}


profile of symptoms has been taken into account. Nearly half of MADD cases were also grouped in this general 'distress' class. The symptom profile of MADD was similar in our survey to that of 'pure' cases of ICD-10 anxiety disorder and depressive episode, but with fewer specific anxiety and depression symptoms, and a lower overall symptom count than in cases of comorbid ICD-10 anxiety and depressive disorder. Mixed presentations may be the norm, at least in the general population. It would seem that designating cases as 'pure' depressive episode or 'pure' anxiety disorder is often a misnomer - symptoms in the other group are present but insufficient to support a diagnosis in that category. These are certainly not distinct conditions; at the very least they should be considered to be closely related disorders with respect to phenomenology. That the other half of MADD cases were grouped into latent classes dominated by non-cases (characterised by a high frequency of symptoms of fatigue, sleep disturbance and worry, but a low frequency of core symptoms of depression and anxiety) should raise some concerns regarding the clinical significance of the condition, particularly as defined using the National Psychiatric Morbidity survey criterion. Clinical significance still needs to be clarified, particularly with respect to external validators, natural history and response to psychological, social and pharmacological interventions. Neither prognosis nor aetiology could be studied in this analysis of cross-sectional survey data. It may be that this process will help us to define the type, severity and combination of symptoms that would merit a diagnosis. Family history (not addressed in this study) might also help to locate MADD with respect to related conditions. For example, a study by Reich suggests that anxious personality disorders may be increased in relatives of people with comorbid anxiety and depression compared with people with 'pure' anxiety disorders. ${ }^{18}$

\section{Criteria for research and clinical practice}

Research into MADD has been hampered by the variety of definitions in use. This may explain the widely varying estimates of prevalence, ${ }^{19-21}$ as well as conflicting findings on the temporal stability of MADD compared with anxiety or depression. ${ }^{22-24}$ The DSM-IV MADD criterion seems to be too restrictive, ${ }^{2}$ whereas that of ICD-10 is insufficiently operationalised. ${ }^{1}$ The criterion used in our analysis simply required a score of 12 or more on the CIS-R psychiatric morbidity scale (as well as the absence of an ICD-10 diagnosis). As we have seen, this did not in practice guarantee the concurrence of specific symptoms of depression and anxiety; indeed, non-specific symptoms predominated. This may have led to our findings overestimating the prevalence of MADD, compared with more restrictive criteria such as those of DSM-IV. The criterion for MADD used in this study was specific to the CIS-R; nevertheless, it did approximate to the definition of MADD as described in ICD-10. There are a wide variety of definitions of MADD currently in use; for example, Tyrer has proposed criteria for 'cothymia' defined as 'the co-occurrence of anxiety (generalised or panic) and depressive symptoms, with 'both anxiety and depressive symptoms normally being present for at least part of the day, on every day, during the last 4 weeks. ${ }^{25}$ Although the operationalised definition of MADD may be problematic, it seems clear that this should be one area to be developed further in the forthcoming revisions of the two main psychiatric classificatory systems (DSM-IV and ICD-10), and further work is needed on the diagnostic validity of MADD.

\section{Dimensional $v$. categorical models of common mental disorder}

We report no effect of diagnostic group (including MADD) on most impact outcomes after adjusting for CIS-R symptom score, but a large independent effect of CIS-R symptom score on all impact measures after adjusting for diagnosis (including MADD). To our knowledge, ours is the first report of its kind examining directly the explanatory power of dimensional $v$. categorical approaches. The superiority of the dimensional perspective was illustrated both by the independent effect of the CIS-R psychological morbidity score after adjusting for diagnosis, and by the much larger population attributable fraction across all healthrelated quality of life outcomes for fifths of the CIS-R psychological morbidity score as compared with diagnostic group. These findings further confirm limitations inherent within purely categorical approaches to classification. ${ }^{10,26,27}$ The validity of these categorisations has been questioned from a number of different perspectives, one of which has been the perceived danger of 'carving nature at the joints. ${ }^{28}$ In practice, however, both approaches have their place. Categorical approaches, based upon operationally defined criteria, provide an essential common language, with demonstrable reliability for clinical practice and research. There is some evidence for the specificity of pharmacological and psychological treatments, and functional neuroimaging and neuroendocrinological evidence supports the notion of a distinct neurobiology for major depression and generalised anxiety disorder. Our findings strongly support the inclusion of a dimensional perspective, without which the population burden of psychological morbidity is markedly underestimated.

\footnotetext{
Jayati Das-Munshi, MRCPsych, David Goldberg, MD, Institute of Psychiatry, King's College London; Paul E. Bebbington, PhD, Department of Mental Health Sciences, University College London; Dinesh K. Bhugra, PhD, Institute of Psychiatry, King's University College London; Dinesh K. Bhugra, PhD, Institute of Psychiatry, King's
College London; Traolach S. Brugha, MD, Department of Health Sciences, University of Leicester; Michael E. Dewey, PhD, Rachel Jenkins, MD, Rob Stewart, MD, Martin Prince, MD, Institute of Psychiatry, King's College London, UK

Correspondence: Dr J. Das-Munshi, Section of Epidemiology, Institute of Psychiatry, King's College London, De Crespigny Park, London SE5 8AF, UK. Email: spsljdm@iop.kcl.ac.uk

First received 7 Feb 2007, final revision 2 Oct 2007, accepted 15 oct 2007
}

\section{Acknowledgements}

This work was developed, in part, to inform the American Psychiatric Institute for Research and Education's Task Force led review of the DSM-IV classification. M.P. and D.G. are members of the Depression and Generalised Anxiety Disorder Working Group. No funding was received. We are grateful to Hans Ormel for helpful comments on an earlier draft of this paper.

\section{References}

1 World Health Organization. The ICD-10 Classification of Mental and Behavioural Disorders: Clinical Descriptions and Diagnostic Guidelines. WHO, 1992.

2 American Psychiatric Association. Diagnostic and Statistical Manual of Mental Disorder (4th edn) (DSM-IV). APA, 1994.

3 Katon W, Roy-Byrne PP. Mixed anxiety and depression. J Abnorm Psychol 1991; 100: 337-45.

4 Overall JE, Hollister LE, Johnson M, Pennington V. Nosology of depression and differential response to drugs. JAMA 1966; 195: 946-8.

5 Paykel ES. Classification of depressed patients: a cluster analysis derived grouping. Br J Psychiatry 1971; 118: 275-88.

6 Singleton N, Bumpstead R, O'Brien M, Lee A, Meltzer H. Psychiatric Morbidity Among Adults Living in Private Households. TSO (The Stationery Office), 2001.

7 Goldberg D, Huxley P. Common Mental Disorders: A Bio-social Model. Routledge/Tavistock, 1992.

8 Goldberg D, Goodyer I. The Origins and Course of Common Mental Disorders. Routledge, 2005.

9 Maj M. 'Psychiatric comorbidity': an artefact of current diagnostic systems? Br J Psychiatry 2005; 186: 182-4 
10 Melzer D, Tom BDM, Brugha TS, Fryers T, Meltzer H. Common mental disorder symptom counts in population: are there distinct case groups above epidemiological cut offs? Psychol Med 2002; 32: 1195-201.

11 Wilson $P$, Elliot $D$. An evaluation of the PAF as a sampling frame and its use within OPCS. J R Stat SOC Series A 1987; 150: 230-40.

12 Kish L. Survey Sampling. Wiley, 1965.

13 Lewis G, Pelosi AJ, Araya R, Dunn G. Measuring psychiatric disorder in the community: a standardized assessment for use by lay interviewers. Psycho Med 1992; 22: 465-86.

14 Babor TF, Higgins-Biddle JC, Saunders JB, Monteiro MG. AUDIT: Alcohol Use Disorders Identification Test: Guidelines For Use in Primary Care (2nd edn). World Health Organization, 2001.

15 Ware JE, Kosinski MMA, Keller SD. A 12-item Short Form Health Survey: construction of scales and preliminary tests of reliability and validity. Med Care 1996; 34: 220-33.

16 Brugha TS, Bebbington $\mathrm{PE}$, Jenkins $\mathrm{R}$. A difference that matters: comparisons of structured and semi-structured psychiatric diagnostic interviews in the general population. Psychol Med 1999; 29: 1013-20.

17 Brugha TS, Jenkins R, Taub N, Meltzer H, Bebbington PE. A general population comparison of the Composite International Diagnostic Interview (CIDI) and the Schedules for Clinical Assessment in Neuropsychiatry (SCAN) Psychol Med 2001; 31: 1001-13.

18 Reich J. Distinguishing mixed anxiety/ depression from anxiety and depressive groups using the family history method. Compr Psychiatr 1993; 34: $285-90$
19 Wittchen HU, Essau CA. Comorbidity and mixed anxiety-depressive disorders: is there epidemiologic evidence? J Clin Psychiatry 1993; 54: 9-15.

20 Sartorius N, Ustun BB, Lecrubier Y, Wittchen HU. Depression comorbid with anxiety: results from the WHO study on psychological disorders in primary health care. Br J Psychiatry 1996; 168 (suppl 30): s38-43.

21 Weisberg RB, Maki KM, Culpepper L, Keller MB. Is anyone really MADD? The occurrence and course of mixed anxiety and depressive disorder in a sample of primary care patients. J Nerv Ment Dis 2005; 193: 223-30.

22 Merikangas KR, Zhang $H$, Avenovoli S, Acharyya S, Neuenschwander M, Angst J. Longitudinal trajectories of depression and anxiety in a prospective community study; the Zurich cohort study. Arch Gen Psychiatry 2003; 60: 993-1000.

23 Usall J, Marquez M. Mixed anxiety and depression disorder: a naturalistic study. Actas Esp Psiquiatr 1999; 27: 81-6.

24 Barkow K, Heun R, Wittchen HU, Ustun TB, Gansicke M, Maier W. Mixed anxiety-depression in a 1-year follow up study: shift to other diagnoses or remission? J Affect Disord 2004; 79: 235-9.

25 Tyrer P. Classification of Neurosis. John Wiley \& Sons, 1989.

26 Katerndahl DA, Larme AC, Palmer RF, Armodei N. Reflections on DSM classification and its utility in primary care: case studies in 'mental disorders'. J Clin Psychiatry 2005; 7: 91-9.

27 Goldberg D. Plato versus Aristotle: categorical and dimensional models for common mental disorders. Compr Psychiatry 2000; 41: 8-13.

28 Krueger RF. The structure of common mental disorders. Arch Gen Psychiatry 1999; 56: 921-6.

\section{War psychiatry}

Simon Wessely

War is hell, but it can be a job - a strange job in which one voluntarily (these days) exposes oneself to the risk of physical and psychiatric injury. Our generation think we discovered post-traumatic stress disorder, but it is neither new, nor the commonest, mental health problem in the UK Armed Forces. That 'honour' goes to depression and alcohol. Are these always the result of going to war? No, things are rarely that simple. Can we treat them? Sometimes - but what makes people good soldiers makes them bad patients. Can we prevent them? Possibly - but only if we don't send people to war. 